\title{
BMJ Open Efficacy of virtual reality techniques in cardiopulmonary resuscitation training: protocol for a meta-analysis of randomised controlled trials and trial sequential analysis
}

Jianqiao Zheng (1) ${ }^{1}$ Li Du, ${ }^{2}$ Xiaoqian Deng, ${ }^{1}$ Lu Zhang, ${ }^{1}$ Jia Wang, ${ }^{1}$ Guo Chen ${ }^{1}$

To cite: Zheng J, Du L, Deng $X$, et al. Efficacy of virtual reality techniques in cardiopulmonary resuscitation training: protocol for a metaanalysis of randomised controlled trials and trial sequential analysis. BMJ Open 2022;12:e058827. doi:10.1136/ bmjopen-2021-058827

- Prepublication history and additional supplemental material for this paper are available online. To view these files, please visit the journal online (http://dx.doi.org/10.1136/ bmjopen-2021-058827).

Received 29 0ctober 2021 Accepted 24 January 2022

D) Check for updates

(c) Author(s) (or their employer(s)) 2022. Re-use permitted under CC BY-NC. No commercial re-use. See rights and permissions. Published by BMJ.

${ }^{1}$ Department of Anesthesiology, West China Hospital, Sichuan University, Chengdu, Sichuan,

China

${ }^{2}$ Department of Anesthesiology, University of Electronic Science and Technology of China, Chengdu, Sichuan, China

Correspondence to

Professor Guo Chen;

Anesthesiology_SCU@163.com

\section{ABSTRACT}

Introduction Cardiopulmonary resuscitation (CPR) is the most critical procedure in the rescue of patients with sudden cardiac death (SCD). The success rate of CPR remains far below expectations, which made CPR education identified as the top priority for SCD. CPR training using the virtual reality (VR) technique is a feasible training method, with a wider population and lower cost, but its efficacy remains controversial. Thus, we will perform a protocol for a systematic review and metaanalysis to identify the efficacy of the VR technique on CPR quality.

Methods and analysis We will search PubMed, Web of Science, Cochrane Library, Ovid Medline, Embase, China National Knowledge Infrastructure, Chinese BioMedical Literature, Wanfang and VIP databases from inception to November 2021, to identify randomised controlled trials and the first period in randomised cross-over trials assessing the efficacy of VR techniques versus non-VR techniques for adult participants accepting adult CPR training. No language restrictions will be considered. Data synthesis will be performed using RevMan V.5.4 and Stata/MP V.16.0. Outcome measures will be present as relative risk with $95 \% \mathrm{Cls}$ for dichotomous data and mean difference with $95 \%$ Cls for continuous data. The primary outcome will be the CPR quality defined as chest compression rate and depth. Secondary outcomes will be the overall performance of CPR. Heterogeneity will be assessed by the $\chi^{2}$ test and $I^{2}$ statistic. Data will be synthesised by either fixed-effects or random-effects models according to the $I^{2}$ value. Trial sequential analysis and modified Jadad Scale will be used to control the risks of random errors and evaluate the evidence quality. Egger's regression test and funnel plots will be used to assess the publication bias.

Ethics and dissemination Ethical approval was not required for this systematic review protocol. The findings will be disseminated through peer-reviewed publications. PROSPERO registration number CRD42021281059.

\section{INTRODUCTION}

Sudden cardiac death is a major global public health problem, accounting for up to $20 \%$ of deaths in Western societies. ${ }^{1}$ It is also one
Strengths and limitations of this study

- This systematic review protocol follows the Preferred Reporting Items for Systematic Reviews and MetaAnalyses Protocols guidelines to conduct a rigorous risk of bias assessment.

- Trial sequential analysis will be used to calculate the required information size for the outcomes to control the risks of false-positive.

- Subgroup analysis will be performed to assess the heterogeneity according to participants' medical background, type of virtual reality (VR) techniques and type of non-VR techniques.

- Publication bias will be assessed by funnel plots and Egger's regression test.

- Limitations will be the heterogeneity caused by the small sample size in each study and the limited number of participants for subgroup analyses.

of the leading causes of death in the USA, with nearly 380000 deaths per year. ${ }^{2}$ Cardiopulmonary resuscitation (CPR) is the most urgent and critical procedure in the rescue of patients with cardiac arrest. ${ }^{3}$ CPR can save lives by artificial ventilation and chest compressions, which preserve circulation to the brain when the cardiac arrest occurred. ${ }^{4-6}$ Early CPR could improve survival in both outof-hospital and in-hospital cardiac arrests. ${ }^{78}$

Despite great efforts that have been made to improve the knowledge of CPR over 50 years, the success rate of CPR remains far below expectations. ${ }^{9}$ Only $11.4 \%$ of patients with out-of-hospital cardiac arrest and $23.8 \%$ of patients with in-hospital cardiac arrest survived even when CPR intervention is performed. ${ }^{10}$ There were approximately $10 \%$ out-of-hospital cardiac arrest survival to hospital discharge in the USA. ${ }^{11}$ Restoring adequate circulation promptly is an important variable determining survival 
in cardiac arrest. ${ }^{12} 13$ Approximate $40 \%$ of cardiac arrest cases unfortunately miss the early CPR. ${ }^{14-20}$ Even within hospitals, high-quality chest compressions are performed in $13 \%-64 \%$ of cardiac arrest cases only. ${ }^{21-23}$ In this situation, leading authorities have identified research on education as one of the top priorities for cardiac arrest research. $^{2425}$

Current guidelines state that high-level scientific evidence on the optimal CPR training method is scarce ${ }^{26}{ }^{27}$ Face-to-face CPR training has always been the gold standard, but developments in new technologies may provide rapid, easily accessible CPR training to a wider population and can be done at home at a low cost. ${ }^{26-28}$ The urgency to reduce group training has increased as trainees are in close contact during most of the current CPR training methods in the recent novel COVID-19 pandemic. Therefore, providing up-to-date CPR training methods for both healthcare professionals and members of the public, which could improve the training quality, is of vital importance.

Virtual reality (VR) is an innovative technology that involves real-time simulation and interactions through visual and auditory sensorial channels created by a computer. ${ }^{29}{ }^{30}$ Trainees can interact with or within environments with enhanced feedback under VR environment. ${ }^{31}{ }^{32}$ It has been considered as potentially a powerful tool to improve interaction and performance with manikin simulators. ${ }^{33} \mathrm{VR}$ technique is an interesting and promising new way expanding quickly in medical education teaching, as its high level of immersion. ${ }^{34}$ In addition, VR technology has also been introduced into clinical practice, its importance and availability have increased significantly. VR technique can improve surgical technical skills, decrease operation time and improve operative performance, which was supported by systematic reviews. ${ }^{36} 37$

CPR training using the VR technique is a feasible and effective training method, which is highly valued by medical students. ${ }^{38}$ VR training can be conducted at home at a low cost at any time and takes a very short time to complete. VR training could overcome important barriers for layperson CPR training. ${ }^{39} 40$ Experts consider the VR technique as one of the most promising tools in medical training, particularly in CPR training. ${ }^{41}{ }^{42} \mathrm{VR}$ training was developed and endorsed by the Resuscitation Council of the UK and is specifically mentioned in current CPR guidelines. ${ }^{26}{ }^{39-42}$ It is reported that VR training resulted in comparable chest compression rate but inferior compression depth compared with faceto-face training. ${ }^{43}$ To improve the training quality, the optimal combination of chest compression depth and rate in VR resuscitation training was proven to be an easily available vector to disseminate CPR skills. ${ }^{44}$

Hence, the efficacy of VR training on CPR quality remains unclear until now. This review aims to review published material on VR technique in CPR training to evaluate its efficacy on CPR quality defined as chest compression depth and compression rate. Outcomes of this systematic review will provide evidence for further research on CPR training education.

\section{Objectives}

We are conducting this protocol of systematic review and meta-analysis to determine the efficacy of VR technique on CPR training quality defined as chest compression depth and compression rate. Furthermore, we will use trial sequential analysis (TSA) to confirm the reliability of the results.

\section{METHODS AND ANALYSIS \\ Study design}

Our review protocol has been registered with PROSPERO (registration number: CRD42021281059). This protocol was prepared under the guidelines of the Preferred Reporting Items for Systematic Reviews and MetaAnalyses Protocols (PRISMA-P) ${ }^{45}$ The systematic review and meta-analysis will be conducted in accordance with the Cochrane Handbook and reported according to the PRISMA statement. ${ }^{46} 47$ The study is expected to begin searching in November 2021 and end in February 2022.

\section{Inclusion/exclusion criteria for study selection \\ Types of studies}

We will include all randomised controlled trials (RCTs) and the first period in randomised cross-over trials involving the efficacy of VR techniques for CPR training. There will be no language restrictions applied.

Studies with the following situations will be excluded: (1) participants accepting children or neonatal CPR training by VR techniques; (2) studies without a control group; (3) studies comparing different types of VR techniques; (4) studies with incorrect data, incomplete data or the research data could not be used for statistical analysis; (5) studies with abstracts from conferences, editorials, duplicate publications, observational studies and retrospective studies.

\section{Types of participants}

Adult participants (age $\geq 18$ years old) accepting adult CPR training by VR techniques will be included. There will be no limits on participants' gender and medical background.

\section{Types of interventions/controls}

In the intervention group, participants had to accept adult CPR training by VR techniques (including the VR mobile application, Lifesaver VR CPR training or the VR scenario of sudden cardiac arrest), then perform the CPR simulation on CPR manikins.

The control group will be the participants who received adult CPR training by non-VR techniques including standardised face-to-face CPR training or standard CPR mobile application video, then perform the CPR simulation on CPR manikins. 
Types of outcome measures

\section{Primary outcomes}

Chest compression rate and depth are the most widely studied CPR quality parameters, with strong associations with patient outcome, which was confirmed by a review on CPR education. ${ }^{48}$ So, the primary outcome will be the CPR quality, expressed as chest compression depth (millimetres) and chest compression rate (per minute), which measured objectively using certified CPR manikins.

\section{Secondary outcomes}

1. Overall performance of CPR.

- Proportions of adequate compression: defined as compression rate between 100 and 120 per min and compression depth between 50 and $60 \mathrm{~mm}$ within guideline ranges. ${ }^{49}$

- Proportions of correct compression rate: defined as compression rate within 100-120 per min meeting the guideline for CPR quality criteria.

- Proportions of correct compression depth: defined as compression depth within 50-60 mm meeting the guideline for CPR quality criteria.

2. Proportions of full chest relaxation (FCR).

After reaching the correct chest compression depth, FCR generates an appropriate pressure difference in the chest, which determines the perfusion pressure. Incomplete chest compression relaxation will reduce perfusion pressure, thereby decreasing the return of spontaneous circulation. $^{50}$ Thus, FCR is an important CPR quality factor. In addition, FCR is the most difficult part for participants to perform within such a short time especially for those training in CPR for the first time. So, FCR will be used as a measure for learning. ${ }^{51}$

3. Chest compression fraction: defined as the proportion of time spent delivering chest compressions during CPR.

\section{Exploratory outcomes}

Exploratory outcomes will be the participants' response data of sudden cardiac arrest, expressed as noticing sudden cardiac arrest, interpreting sudden cardiac arrest as a problem, calling 911, performing CPR and asking for an automated external defibrillator.

\section{Search strategy}

English and Chinese electronic databases from inception to November 2021 will be searched for published literature. English databases include Cochrane Library, PubMed, Web of Science, Ovid MEDLINE and Embase. Chinese databases include Chinese BioMedical Literature, China National Knowledge Infrastructure, VIP database and Wanfang database. Reference lists of each literature and trial registry database (WHO International Clinical Trials Registry Platform and ClinicalTrials.gov) will also be scrutinised for missing studies and unpublished or ongoing clinical trials. After data extraction, corresponding authors of the included literature will be asked for more related original data to avoid potential missing as much as possible.

The search strategy for PubMed is shown in table 1 as an example. The following search terms will be used: virtual reality, augmented reality, cardiopulmonary resuscitation and randomized controlled trial. The search terms will be translated into Chinese for study identification in Chinese databases. We will perform a new search in the databases to check if any studies were published during the elaboration of the systematic review before the final publication. The preliminary search strategy is given in online supplemental file 1 .

\section{Data collection and analysis}

Selection of studies

Two reviewers (JZ and JW) will be required to screen the retrieved studies independently. Briefly, they will exclude

\begin{tabular}{|c|c|}
\hline No & Search terms \\
\hline \#1 & $\begin{array}{l}\text { "virtual reality" [MeSH] OR"augmented reality" [MeSH] OR "user-computer interface" [MeSH] OR "Computer } \\
\text { Simulation"[MeSH] OR "Video Game" [MeSH] OR "augmented reality" [MeSH] OR (virtual* OR simulat*)[Title/ } \\
\text { Abstract]OR "virtual reality"[Title/Abstract]OR "augmented reality"[Title/Abstract]OR "user-computer interface"[Title/ } \\
\text { Abstract]OR "Video Game"[Title/Abstract]OR "augmented reality"[Title/Abstract] }\end{array}$ \\
\hline \#2 & $\begin{array}{l}\text { “Lifesaver VR"[Title/Abstract]OR “Mini-VREM”[Title/Abstract]OR “Virtual CPR”[Title/Abstract]OR “VR ACT”[Title/ } \\
\text { Abstract] }\end{array}$ \\
\hline \#3 & \#1 OR \#2 \\
\hline \#4 & $\begin{array}{l}\text { "Heart Arrest" [MeSH] OR "Cardiopulmonary Resuscitation" [MeSH] OR "Sudden Cardiac Arrest" [MeSH] OR " } \\
\text { Sudden Cardiac Death "[MeSH] OR "Cardiac Sudden Death" [MeSH] OR "Cardiac Arrest, Sudden" [MeSH] OR } \\
\text { (Heart Arrest)[Title/Abstract]OR "Cardiopulmonary Resuscitation"[Title/Abstract]OR "Sudden Cardiac Arrest"[Title/ } \\
\text { Abstract]OR "Sudden Cardiac Death"[Title/Abstract]OR "Cardiac Sudden Death"[Title/Abstract]OR "Cardiac Arrest, } \\
\text { Sudden"[Title/Abstract] }\end{array}$ \\
\hline \#7 & random*[Title/Abstract]OR blind*[Title/Abstract]OR singleblind*[Title/Abstract]OR doubleblind*[Title/Abstract] \\
\hline \#8 & \#3 AND \#6 AND \#7 \\
\hline
\end{tabular}




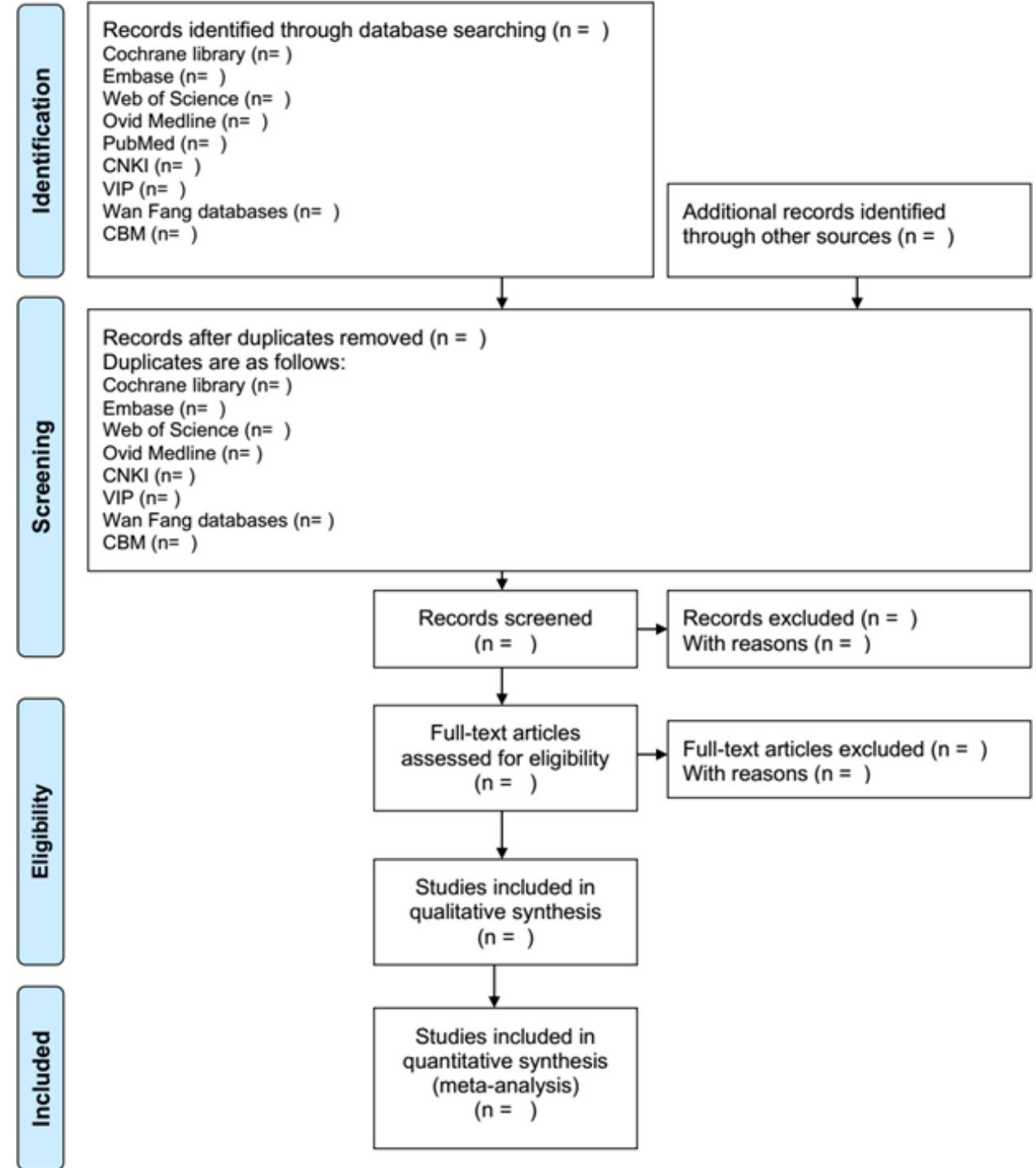

Figure 1 The PRISMA flow diagram. PRISMA, Preferred Reporting Items for Systematic Reviews and Meta-Analyses.

duplicate studies and those not matching the inclusion criteria by reading titles and abstracts. After reading the full text of each study, studies meeting the inclusion criteria will be selected. Any disagreements will be resolved through discussion with a third reviewer (LD). A fourth reviewer (GC) will check all procedures before approving the data extraction. Details of the entire study selection procedure will be shown in the PRISMA flow diagram (figure 1).

\section{Data extraction}

Two reviewers (JZ and LZ) will extract data from the included studies independently following a data acquisition using Microsoft Excel software. Required information includes demographic data, type of VR techniques and non-VR techniques, details of VR and non-VR training, inclusion/exclusion criteria, outcome indicators (primary, secondary and exploratory outcomes), etc. Information about study design (such as randomisation, allocation concealment, blinding methods, data collection and statistical analysis, outcome reporting) will also be recorded for the next step of quality assessment. Results data will be recorded as mean \pm SD for a continuous variable, and the proportion of participants with percentage for dichotomous data. If necessary, a third reviewer (XD) will double-check the data to ensure consistency. If information and data are missing or incomplete in any study, we will contact the corresponding authors of the literature to obtain the original data by email. If necessary, we will extract numerical data from graphs using Adobe Photoshop described by Gheibi $e t a l .{ }^{52}$ Detailed list of information and data to be extracted is presented in table 2.

\section{Quality assessment}

Two reviewers (JW and LZ) will assess the risk of bias in the included studies with the guidance of the Cochrane Handbook independently. ${ }^{53}$ The Cochrane Collaboration's tool covers six aspects: randomisation; allocation concealment; blinding (including blinding of participants and personnel; blinding of outcome assessment); data collection and statistical analysis; selective reporting and other bias. The risk will be divided into three levels 
Table 2 Data and information extraction schedule

\begin{tabular}{ll}
\hline Subject & Content \\
\hline $\begin{array}{l}\text { Publication } \\
\text { information }\end{array}$ & $\begin{array}{l}\text { Name of the first author; contact email; } \\
\text { publication year; country; corporate } \\
\text { sponsorship }\end{array}$ \\
\hline Participant & $\begin{array}{l}\text { Sample size; average age; participants' } \\
\text { medical background (including bystanders, } \\
\text { medical students, medical staff: nurse or } \\
\text { doctor); inclusion and exclusion criteria }\end{array}$ \\
Intervention & $\begin{array}{l}\text { Type of VR techniques; details of VR training } \\
\text { (including training time, equipment for VR }\end{array}$ \\
training and training items)
\end{tabular}

CPR, cardiopulmonary resuscitation; VR, virtual reality.

(low risk, unclear and high risk) in accordance with the item in the checklist. If any disagreements arise, the risk assignment will be settled through discourse. If this discourse is not conducive, discrepancies will be resolved by a third reviewer (GC).

\section{Evidence grade evaluation}

We will apply the modified Jadad Scale to evaluate the quality of each outcome's evidence grade. ${ }^{54}$ Evidence grade evaluation of the included studies will be conducted in eight items: randomisation (with score 0-2), blinding (with score 0-2), withdrawals and dropouts (with score $0-1$ ), inclusion and exclusion criteria (with score $0-1$ ), adverse effects (with score 0-1) and statistical analysis (with score 0-1) (table 3). Scale scores for each study could range from 0 to 8 points, with higher scores indicating better quality: score 1-3 signified low quality; score 4-8 signified high quality.

\section{Measures of treatment effect}

For continuous outcome data, the mean differences (MDs) or the standardised MDs with 95\% CIs will be used for analysis. For dichotomous data, the relative risks (RRs) with $95 \%$ CIs will be used for analysis.
Table 3 The modified Jadad Scale

\begin{tabular}{lc}
\hline Items & Score \\
\hline 1. Was the study described as randomised? & \\
Yes & 1 \\
No & 0
\end{tabular}

2. Was the method of randomisation appropriate?

$\begin{array}{lc}\text { Yes } & 1 \\ \text { No } & -1 \\ \text { Not described } & 0 \\ \text { 3. Was the study described as blinded? } \\ \text { Yes (double-blind) } \\ \text { Yes (single-blind) } & 1 \\ \text { No } & 0.5\end{array}$

4. Was the method of blinding appropriate?

$\begin{array}{lc}\text { Yes } & 1 \\ \text { No } & -1 \\ \text { Not described } & 0\end{array}$

5. Was there a description of withdrawals and dropouts?

$\begin{array}{ll}\text { Yes } & 1 \\ \text { No } & 0 \\ \begin{array}{l}\text { 6. Was there a clear description of the inclusion } \\ \text { and exclusion criteria? }\end{array} \\ \begin{array}{ll}\text { Yes } & 1 \\ \text { No } & 0\end{array}\end{array}$

7. Was the method used to assess adverse effects described?

\begin{tabular}{ll} 
Yes & 1 \\
No & 0 \\
$\begin{array}{l}\text { 8. Was the method of statistical analysis } \\
\text { described? }\end{array}$ \\
Yes & 1 \\
No & 0 \\
\hline
\end{tabular}

\section{Assessment of heterogeneity}

We will calculate $\mathrm{I}^{2}$ to test heterogeneity for each pooled result with Review Manager V.5.4 (RevMan, Cochrane Collaboration, Oxford, UK). Statistical heterogeneity will be assessed by the standard $\chi^{2}$ test $(\alpha=0.1)$ and $\mathrm{I}^{2}$ test. If $\mathrm{p} \geq 0.1$ and if $\mathrm{I}^{2} \leq 50 \%$, a fixed-effects model will be used. If $\mathrm{p}<0.1$ or $\mathrm{I}^{2}>50 \%$, random-effects models will be applied. When the heterogeneity is statistically significant, we will conduct a subgroup analysis to investigate the possible sources of heterogeneity according to the participants' medical background, type of VR techniques and type of non-VR techniques. If $\mathrm{I}^{2}>75 \%$, a meta-analysis will not be performed and a narrative, a qualitative summary will be provided.

\section{Trial sequential analysis}

TSA will be performed by Stata/MP V.16.0 (Stata Corp, College Station, Texas, USA) to control the risks of 
false-positive by calculating the required information size (RIS) ${ }^{55}$ RIS is the number of participants needed in a meta-analysis to detect or reject a certain intervention effect. ${ }^{56}$ RIS and information size for primary and secondary outcomes will be calculated. In addition, the cumulative Z-curve's breach of relevant trial sequential to monitor boundaries will be calculated for all outcomes.

For continuous outcomes, we will use the observed SD, an MD of the observed SD/2, an alpha of $5 \%$ and a beta of $90 \%$ for primary and secondary outcomes in the TSA. ${ }^{57}$ For dichotomous outcomes, we will use the proportion of participants with an outcome in the control group, an RR increased 0.10, and an alpha of 0.05 and a beta of 0.90 in the TSA. ${ }^{58}$ TSA will be performed using the TSA program V.0.9.5.10 Beta (http://www.ctu.dk/tsa). ${ }^{59}$

\section{Subgroup analysis}

We will further explain the results with an analysis of subgroups or subsets. If sufficient trials are available, data from participants with different medical backgrounds, different types of VR techniques and non-VR techniques will be analysed separately.

- Participants' medical background (including bystanders, medical students, medical staff: nurse or doctor).

- Different types of VR techniques (including VR mobile application, Lifesaver VR CPR training or the VR scenario of sudden cardiac arrest).

- Different types of non-VR techniques (including standardised face-to face CPR training or standard CPR mobile application video).

\section{Sensitivity analysis}

Sensitivity analysis will be used to evaluate how uncertain assumptions of data and usage affect the robustness of the combined results. Low-quality studies will be excluded; we will reanalyse the included studies and assess whether there are significant differences between the combined effects. If necessary, the included studies will be removed one by one, then we will observe whether the pooled estimations are stable or not. Significant changes may indicate significant heterogeneity among studies.

\section{Assessment of publication biases}

The potential publication bias will be statistically analysed by funnel plot analysis and Egger's regression test while no less than 10 original studies are involved for an outcome. ${ }^{6061}$ The trim-and-fill analysis will also be done to adjust any potential publication bias, as it is based on the assumption that the effect sizes of all the studies are normally distributed around the centre of a funnel plot in the absence of publication bias. ${ }^{62}$ Publication biases will be performed by Stata/MP V.16.0.

\section{Patient and public involvement}

Patients or the public were not involved in the design, or conduct, or reporting, or dissemination plans of our research.
Ethics and dissemination

Ethical approval was not required for this systematic review protocol. The findings will be disseminated through peerreviewed publications.

\section{DISCUSSION}

Cardiac arrest is a significant public health issue, as it has low survival rates and a high risk for irreversible neurological damage in survivors. Bystanders witness approximately $50 \%$ of out-of-hospital cardiac arrests. ${ }^{63}$ Unfortunately, most bystanders did not perform any form of CPR, as they are not currently trained in CPR training. ${ }^{64}$ Even within hospitals, high-quality chest compressions do not occur in $36 \%-87 \%$ of cardiac arrest cases. ${ }^{21-23}$ It is estimated that more than 100000 deaths will be saved and neurologically intact survival would increase up to fourfold, if the general public were educated and engaged in performing CPR. ${ }^{63}$ Hence, how to improve the quality of CPR on the out-of-hospital and in-hospital cardiac arrests by most efficient training technique is promoted as a key issue. Creating awareness, increasing willingness, engaging the general public in training and capability of civilians to perform high-quality CPR are important.

VR has shown great potential in the area of CPR, especially gatherings of large groups or close encounters are strongly discouraged in the context of the COVID-19 pandemic. CPR training by VR may provide important insights on potential solutions to overcome these challenges, and enhance CPR training quality. The aim of the study will provide an overview of the current state of evidence concerning the efficacy of VR training to enhance CPR training quality. We will evaluate the effect of CPR training quality by chest compression depth and rate. In addition, we will investigate the efficacy of VR training on the overall performance of CPR, the full chest relaxation, the chest compression fraction and participants' response data to sudden cardiac arrest. To the best of our knowledge, this will be the first systematic review concerning this topic. Outcomes of this systematic review will provide evidence for updating the VR training methods and then improving the CPR quality provided by healthcare professionals and the general public.

This systematic review protocol follows the PRISMA-P guidelines. Strengths of our systematic review include the following: first, comprehensive search in English and Chinese databases. Second, multivariable analysis (including assessment of study quality, subgroup analysis, sensitivity analysis, TSA and Egger's regression test) will be used to minimise the confounding bias. Third, screening, data extraction and quality assessment will be performed by two independent reviewers according to guidelines.

There are also limitations to our analysis. First, studies with different VR techniques and non-VR techniques, participants with different medical backgrounds and different types of CPR manikins will be included, resulting in potential heterogeneity. Second, the number of studies with eligible data for subgroup analyses may be limited. 
Third, the sample size in each study may be small. Fourth, articles that met inclusion criteria might be missed during the electronic search, as the keywords for new VR techniques may change quickly in the future. Furthermore, another limitation may be the current lack of high-level evidence, such as well-designed RCTs which are double blind. Thus, we will use rigorous methods such as the TSA and trim-and-fill analysis in the data analysis and will meta-analyse the outcomes as appropriate.

\section{Timeline}

Formal screening of search results will begin in November 2021. Data extraction will begin in December 2021. The project is due to complete in February 2022.

Contributors $\mathrm{JZ}$ and $\mathrm{LD}$ conceived the idea for this systematic review. All authors (JZ, LD, JW, LZ, XD, GC) developed the methodology for the systematic review. The manuscript was drafted by JZ and LD, and revised by all authors. XD and GC will screen potential studies and perform duplicate independent data abstraction. JZ and $L Z$ will undertake the risk of bias assessment and assess the evidence quality. JZ and LD will conduct the data synthesis. All authors contributed to the research and agreed to be responsible for all aspects of the work.

Funding The authors have not declared a specific grant for this research from any funding agency in the public, commercial or not-for-profit sectors.

Competing interests None declared.

Patient consent for publication Not required.

Provenance and peer review Not commissioned; externally peer reviewed.

Supplemental material This content has been supplied by the author(s). It has not been vetted by BMJ Publishing Group Limited (BMJ) and may not have been peer-reviewed. Any opinions or recommendations discussed are solely those of the author(s) and are not endorsed by BMJ. BMJ disclaims all liability and responsibility arising from any reliance placed on the content. Where the content includes any translated material, BMJ does not warrant the accuracy and reliability of the translations (including but not limited to local regulations, clinical guidelines, terminology, drug names and drug dosages), and is not responsible for any error and/or omissions arising from translation and adaptation or otherwise.

Open access This is an open access article distributed in accordance with the Creative Commons Attribution Non Commercial (CC BY-NC 4.0) license, which permits others to distribute, remix, adapt, build upon this work non-commercially, and license their derivative works on different terms, provided the original work is properly cited, appropriate credit is given, any changes made indicated, and the use is non-commercial. See: http://creativecommons.org/licenses/by-nc/4.0/.

ORCID iD

Jianqiao Zheng http://orcid.org/0000-0002-8091-1837

\section{REFERENCES}

1 Wong CX, Brown A, Lau DH, et al. Epidemiology of sudden cardiac death: global and regional perspectives. Heart Lung Circ 2019;28:6-14.

2 Virani SS, Alonso A, Benjamin EJ, et al. Heart disease and stroke Statistics-2020 update: a report from the American heart association. Circulation 2020;141:e139-596.

3 Blewer AL, Leary M, Esposito EC, et al. Continuous chest compression cardiopulmonary resuscitation training promotes rescuer self-confidence and increased secondary training: a hospitalbased randomized controlled trial*. Crit Care Med 2012;40:787-92.

4 Kouwenhoven WB, Jude JR, Knickerbocker GG. Closed-Chest cardiac massage. JAMA 1960;173:1064-7.

5 Herlitz J, Ekström L, Wennerblom B, et al. Effect of bystander initiated cardiopulmonary resuscitation on ventricular fibrillation and survival after witnessed cardiac arrest outside Hospital. Br Heart J 1994;72:408-12.

6 Noordergraaf GJ, Ottesen JT, Scheffer GJ. Cardiopulmonary resuscitation: biomedical and biophysical analysis. In: Biomedical engineering Handbook. Vol 33. 3 edn, 2004.
7 Hasselqvist-Ax I, Riva G, Herlitz J, et al. Early cardiopulmonary resuscitation in out-of-hospital cardiac arrest. $N$ Engl J Med Overseas Ed 2015;372:2307-15.

8 Ornato JP, Peberdy MA, Reid RD, et al. Impact of resuscitation system errors on survival from in-hospital cardiac arrest. Resuscitation 2012;83:63-9.

9 Eisenberg MS, Mengert TJ. Cardiac resuscitation. N Engl J Med 2001;344:1304-13.

10 Benjamin EJ, Blaha MJ, Chiuve SE, et al. Heart disease and stroke statistics-2017 update: a report from the American heart association. Circulation 2017;135:e146-603.

11 Virani SS, Alonso A, Aparicio HJ, et al. Heart disease and stroke Statistics-2021 update: a report from the American heart association. Circulation 2021;143:e254-43.

12 Friess SH, Sutton RM, French B, et al. Hemodynamic directed CPR improves cerebral perfusion pressure and brain tissue oxygenation. Resuscitation 2014;85:1298-303.

13 Everly GS, Flynn BW. Principles and practical procedures for acute psychological first aid training for personnel without mental health experience. Int J Emerg Ment Health 2006;8:93-100.

14 Gräsner J-T, Wnent J, Herlitz J, et al. Survival after out-of-hospital cardiac arrest in Europe - Results of the EuReCa TWO study. Resuscitation 2020;148:218-26.

15 Nas J, Thannhauser J, Herrmann JJ, et al. Changes in automated external defibrillator use and survival after out-of-hospital cardiac arrest in the Nijmegen area. Neth Heart J 2018;26:600-5.

16 Sasson C, Rogers MAM, Dahl J, et al. Predictors of survival from outof-hospital cardiac arrest: a systematic review and meta-analysis. Circ Cardiovasc Qual Outcomes 2010;3:63-81.

17 Rajan S, Wissenberg M, Folke F, et al. Association of bystander cardiopulmonary resuscitation and survival according to ambulance response times after out-of-hospital cardiac arrest. Circulation 2016;134:2095-104.

18 Hasselqvist-Ax I, Riva G, Herlitz J, et al. Early cardiopulmonary resuscitation in out-of-hospital cardiac arrest. $N$ Engl J Med 2015;372:2307-15.

19 Kragholm K, Wissenberg M, Mortensen RN, et al. Bystander efforts and 1-year outcomes in out-of-hospital cardiac arrest. N Engl J Med 2017;376:1737-47

20 Malta Hansen C, Kragholm K, Pearson DA, et al. Association of bystander and First-Responder intervention with survival after out-of-hospital cardiac arrest in North Carolina, 2010-2013. JAMA 2015;314:255-64.

21 Sutton RM, Wolfe $\mathrm{H}$, Nishisaki $\mathrm{A}$, et al. Pushing harder, pushing faster, minimizing interruptions... but falling short of 2010 cardiopulmonary resuscitation targets during in-hospital pediatric and adolescent resuscitation. Resuscitation 2013;84:1680-4.

22 Cheng A, Brown LL, Duff JP, et al. Improving cardiopulmonary resuscitation with a CPR feedback device and refresher simulations (CPR cares study): a randomized clinical trial. JAMA Pediatr 2015;169:137-44.

23 Sutton RM, Niles D, Nysaether J, et al. Quantitative analysis of CPR quality during in-hospital resuscitation of older children and adolescents. Pediatrics 2009;124:494-9.

24 Søreide E, Morrison L, Hillman K, et al. The formula for survival in resuscitation. Resuscitation 2013;84:1487-93.

25 Panchal AR, Cash RE, Crowe RP, et al. Delphi analysis of science gaps in the 2015 American heart association cardiac arrest guidelines. J Am Heart Assoc 2018;7:e008571.

26 Wyckoff MH, Singletary EM, Soar J, et al. 2021 international consensus on cardiopulmonary resuscitation and emergency cardiovascular care science with treatment recommendations: summary from the basic life support; advanced life support; neonatal life support; education, implementation, and teams; first aid task forces; and the COVID-19 Working group. Resuscitation 2021;169:229-311.

27 Greif R, Lockey A, Breckwoldt J, et al. European resuscitation Council guidelines 2021: education for resuscitation. Resuscitation 2021;161:388-407.

28 Yeung J, Kovic I, Vidacic M, et al. The school lifesavers study-A randomised controlled trial comparing the impact of lifesaver only, face-to-face training only, and lifesaver with face-to-face training on CPR knowledge, skills and attitudes in UK school children. Resuscitation 2017;120:138-45.

29 Mazurek J, Kiper P, Cieślik B, et al. Virtual reality in medicine: a brief overview and future research directions. $\mathrm{Hm}$ 2019;20:16-22.

30 Rutkowski S, Rutkowska A, Jastrzębski D, et al. Effect of virtual Reality-Based rehabilitation on physical fitness in patients with chronic obstructive pulmonary disease. J Hum Kinet 2019;69:149-57. 
31 Kiper P, Szczudlik A, Mirek E, et al. The application of virtual reality in neuro-rehabilitation: motor re-learning supported by innovative technologies. Med Rehabil 2014;17:29-36.

32 Rutkowski S, Rutkowska A, Kiper P, et al. Virtual reality rehabilitation in patients with chronic obstructive pulmonary disease: a randomized controlled trial. Int J Chron Obstruct Pulmon Dis 2020;15:117-24

33 Haans A, IJsselsteijn W. Mediated social touch: a review of current research and future directions. Virtual Real 2006;9:149-59.

34 Chaballout B, Molloy M, Vaughn J, et al. Feasibility of augmented reality in clinical simulations: using Google glass with manikins. JMIR Med Educ 2016;2:e2.

35 Ingrassia PL, Mormando G, Giudici E, et al. Augmented reality learning environment for basic life support and defibrillation training: usability study. J Med Internet Res 2020;22:e14910.

36 Aïm F, Lonjon G, Hannouche D, et al. Effectiveness of virtual reality training in orthopaedic surgery. Arthroscopy 2016;32:224-32.

37 Nagendran M, Gurusamy KS, Aggarwal R. Virtual reality training for surgical trainees in laparoscopic surgery. Cochrane Database Syst Rev 2013;2013:CD006575.

38 Jaskiewicz F, Kowalewski D, Starosta K, et al. Chest compressions quality during sudden cardiac arrest scenario performed in virtual reality: a crossover study in a training environment. Medicine 2020;99:e23374.

39 Sasson C, Haukoos JS, Bond C, et al. Barriers and facilitators to learning and performing cardiopulmonary resuscitation in neighborhoods with low bystander cardiopulmonary resuscitation prevalence and high rates of cardiac arrest in Columbus, $\mathrm{OH}$. Circ Cardiovasc Qual Outcomes 2013;6:550-8.

40 Wong MAME, Chue S, Jong M, et al. Clinical instructors' perceptions of virtual reality in health professionals' cardiopulmonary resuscitation education. SAGE Open Med 2018;6:2050312118799602.

41 Semeraro F, Scapigliati A, Ristagno G, et al. Virtual Reality for CPR training: How cool is that? Dedicated to the "next generation". Resuscitation 2017;121:e1-2.

42 Yeung J, Kovic I, Vidacic M, et al. The school lifesavers study-A randomised controlled trial comparing the impact of lifesaver only, face-to-face training only, and lifesaver with face-to-face training on CPR knowledge, skills and attitudes in UK school children. Resuscitation 2017;120:138-45.

43 Nas J, Thannhauser J, Vart P, et al. Effect of face-to-face vs virtual reality training on cardiopulmonary resuscitation quality: a randomized clinical trial. JAMA Cardiol 2020;5:328-35.

44 Nas J, Thannhauser J, van Geuns R-JM, et al. Optimal combination of chest compression depth and rate in virtual reality resuscitation training: a post hoc analysis of the randomized Lowlands saves lives trial. J Am Heart Assoc 2021;10:e017367.

45 Shamseer L, Moher D, Clarke M, et al. Preferred reporting items for systematic review and meta-analysis protocols (PRISMA-P) 2015: elaboration and explanation. BMJ 2015;350:g7647.

46 Higgins JPT TJ, Chandler J, Cumpston M. Cochrane Handbook for systematic reviews of interventions version 6.1 (updated September 2020). The Cochrane collaboration, 2020. www.training. cochrane. org/ handbook

47 Liberati A, Altman DG, Tetzlaff J, et al. The PRISMA statement for reporting systematic reviews and meta-analyses of studies that evaluate healthcare interventions: explanation and elaboration. BMJ 2009;339:b2700.
48 Considine J, Gazmuri RJ, Perkins GD, et al. Chest compression components (rate, depth, chest wall recoil and leaning): a scoping review. Resuscitation 2020;146:188-202.

49 Olasveengen TM, Semeraro F, Ristagno G, et al. European resuscitation Council guidelines 2021: basic life support. Resuscitation 2021;161:98-114.

50 Majer J, Jaguszewski MJ, Frass M, et al. Does the use of cardiopulmonary resuscitation feedback devices improve the quality of chest compressions performed by doctors? A prospective, randomized, cross-over simulation study. Cardiol J 2019;26:529-35.

51 Tanaka S, Tsukigase K, Hara T, et al. Effect of real-time visual feedback device 'Quality Cardiopulmonary Resuscitation (QCPR) Classroom' with a metronome sound on layperson CPR training in Japan: a cluster randomized control trial. BMJ Open 2019;9:e026140.

52 Gheibi S, Mahmoodzadeh A, Kashfi K, et al. Data extraction from graphs using Adobe Photoshop: applications for meta-analyses. Int J Endocrinol Metab 2019;17:e95216.

53 Higgins JPT, Altman DG, Gøtzsche PC, et al. The Cochrane collaboration's tool for assessing risk of bias in randomised trials. BMJ 2011;343:d5928.

54 Jadad AR, Moore RA, Carroll D, et al. Assessing the quality of reports of randomized clinical trials: is blinding necessary? Control Clin Trials 1996;17:1-12.

55 Wetterslev J, Jakobsen JC, Gluud C. Trial sequential analysis in systematic reviews with meta-analysis. BMC Med Res Methodol 2017;17:39

56 Imberger G, Thorlund K, Gluud C, et al. False-Positive findings in Cochrane meta-analyses with and without application of trial sequential analysis: an empirical review. BMJ Open 2016;6:e011890.

57 Norman GR, Sloan JA, Wyrwich KW. Interpretation of changes in health-related quality of life: the remarkable universality of half a standard deviation. Med Care 2003;41:582-92.

58 Castellini G, Nielsen EE, Gluud C. Comment on: "Cell therapy for heart disease: Trial sequential analyses of two cochrane reviews". Clin Pharmacol Ther 2017;102:21-4.

59 Thorlund K, Engstrøm J, Wetterslev J. User manual for trial sequential analysis (TSA) Copenhagen trial unit, centre for clinical intervention research, Denmark, 2011. Available: http://www.ctu.dk/ tsa

60 Egger M, Davey Smith G, Schneider M, et al. Bias in meta-analysis detected by a simple, graphical test. BMJ 1997;315:629-34.

61 Sterne JA, Gavaghan D, Egger M. Publication and related bias in meta-analysis: power of statistical tests and prevalence in the literature. J Clin Epidemiol 2000;53:1119-29.

62 Mavridis D, Salanti G. How to assess publication bias: funnel plot, trim-and-fill method and selection models. Evid Based Ment Health 2014;17:30.

63 London ambulance service NHS trust cardiac arrest annual report 2018/19. clinical audit and research unit, London ambulance service NHS trust, 2019. Available: https://www.londonambulance.nhs.uk/ wp-content/uploads/2020/01/Cardiac-Arrest-Annual-Report-20182019.pdf

64 Duval S, Pepe PE, Aufderheide TP, et al. Optimal combination of compression rate and depth during cardiopulmonary resuscitation for functionally favorable survival. JAMA Cardiol 2019;4:900-8.

65 Kragholm K, Wissenberg M, Mortensen RN, et al. Bystander efforts and 1-year outcomes in out-of-hospital cardiac arrest. N Engl J Med 2017;376:1737-47. 